\title{
THE PRINCIPLES OF CHEMOTHERAPY
}

\author{
By C. A. Keele, M.D., F.R.C.P.
}

Rexder in Pharmacology and Therapeutics, Middlesex Hospital Medical School

This introduction to the collected articles on chemotherapy which have appeared recently in the Postgraduate Medical Journal, is intended to deal only with general aspects of the subject. Some of the principles will doubtless appear very obvious, but there is perhaps no harm in repetition concerning such an important branch of medical science. No references to the literature are given here as they are provided by individual authors in their articles.

As in the therapeutic use of any drug, there is really only one aim in chemotherapy, namely, to choose the appropriate drug for the purpose and to administer it in such a way and for such time as are necessary for it to bring about the desired actions without producing unwanted side-effects.

\section{Selection of the Appropriate Drug}

In selecting drugs for the treatment of infections due to invading parasites the guiding principle is 'selective toxicity,' which means that one wants to choose drugs which are maximally harmful to the parasite and at the same time minimally harmful to the infected patient. This factor, first so clearly enunciated by Ehrlich, is fundamental in selecting new chemical substances for therapeutic use. Innumerable compounds which can kill off parasites in vitro are of no therapeutic value because effective parasiticidal doses are also toxic to the cells of the host.

Thus advances in chemotherapy depend on the discovery of drugs which are increasingly selective in poisoning pathogenic parasites. Consider the case of syphilis. Mercury, which was used from early in the $15^{\text {th }}$ century until quite recently, had little if any spirochaeticidal action and readily produced toxic actions; the organic arsenicals, introduced by Ehrlich, are very potent spirochaeticidal agents but are sometimes rather toxic to the patient; bismuth is less toxic than the arsenicals, but has a weaker, though more pro- longed, action on the Treponema pallidum. How- N ever, the combined arsenical-bismuth treatment $\omega$ was undoubtedly an effective regime with a cure $\mathcal{O}$ rate of about 90 per cent. in patients with early $\mathbb{E}^{\omega}$ syphilis who completed the long course of treat- $~$ ment. But the position has now been radically changed, and, as Dr. R. R. Willcox has stated, $\vec{c}$ penicillin is now first choice for the treatment of all stages of syphilis. It may not be quite so toxic $\bar{\nabla}$ to the spirochaete as the organic arsenicals, but $i$ t $\vec{c}$ is far less harmful to the infected patient. Ios favourable ' therapeutic ratio' (the minimum dose which produces toxic effects/the dose which prô duces a full therapeutic effect) means that penicillin may be given in curative doses with far less likelihood of serious toxic complications.

In some instances it is important to remember $\stackrel{\square}{\rightarrow}$ that the various stages of development of $\mathrm{a} \overrightarrow{\mathrm{O}}$ parasite are differentially affected by drugs and Prof. Murgatroyd has discussed this question in relation to the treatment of malaria. The actions of proguanil (paludrine) on the pre-erythrocytic을 parasites of certain sträins of P. falciparum; the schizonticidal actions of quinine, mepacrine, 3 . chloroquine and proguanil, and the gametocidalio actions of pamaquine and pentaquine illustrate $₹$ such differences in sensitivity to drugs of the different stages of the malarial parasite. In amoebic dysentery, too, the choice of drug de-음 pends on the phase of infection. As Dr. Adams points out, emetine hydrochloride is still best for the treatment of the acute attack or complications, N whereas there are many drugs, including aureo-N mycin, which may help in the control of chronic ${ }_{0}^{\omega}$
amoebiasis.

One should add that an acute infection, or the early stages of what may become a chronic one, is always easier to eradicate than a chronic infection. 0 This is probably due to the development of tissueochanges which may be irreversible and which may make the infecting organisms less accessible toके 
drugs. The early stages of syphilis and the more acute forms of tuberculosis are much more susceptible to chemotherapy than the later stages of these diseases.

In bacterial chemotherapy there have been so many advances since the introduction of the sulphonamides in 1935 that the problem often is to decide which of perhaps three or four drugs is the hest for a given infection. In many cases there have not been sufficiently thorough clinical trials to justify a final judgment, but there are certain points which can be made.

The antibiotics and other powerful antibacterial drugs should only be given when there are clear indications for their use. The micro-organisms susceptible to these drugs are now well known and their presence may justifiably be presumed on clinical grounds in many instances, but where there is any doubt the appropriate bacteriological investigations should be undertaken. It must be emphasized that these drugs are inactive against nearly all virus infections. There is therefore no point in giving them for the treatment of influenza, unless, as Dr. Joules has stressed, staphylococcal pneumonia has supervened, when penicillin would be the drug of choice.

There may be certain strains of an organism which are abnormally resistant to drugs. This may occur primarily as, for example, with streptococcus viridans, or it may be the consequence of drug administration, when the term : acquired drug resistance' is used. Drug resistance can develop either during the treatment of an individual infection, e.g. the resistance of M. tuberculosis towards streptomycin, or over a long period a particular bacterial species can become increasingly resistant to a drug to which it was originally very sensitive, e.g. sulphonamides and the gonococcus, and penicillin and Staphylococcus aureus.

The occurrence of drug-resistant organisms is becoming less serious now that there are alternatives to the original drugs of choice. The sulphonamides have been replaced by penicillin in the treatment of gonorrhoea, and infections due to penicillin-resistant staphylococci respond to aureomycin; in the treatment of tuberculosis, streptomycin is now given together with p-aminosalicylic acid, which greatly reduces the likelihood of the development of streptomycin-resistant organisms. However, as Dr. Scadding points out, the development of resistance to streptomycin still restricts the value of this drug in the treatment of the chronic fibrotic forms of pulmonary tuberculosis.

\section{Combined Chemotherapy}

The question as to whether two drugs are better than one is obviously important. In some instances a combined attack is useful; for ex- $\frac{0}{0}$ ample, in pneumococcal meningitis the administra-气 tion of sulphonamides to reach the infected area 3 via the blood stream plus intrathecal penicillin $\stackrel{\mathbb{Q}}{2}$ gives better results than follow the use of either $\stackrel{c}{.}$ drug alone. Penicillin and streptomycin, which $\overrightarrow{\vec{F}}$ both have bactericidal actions, may also act synergistically on organisms susceptible to both; the conjoint actions of streptomycin and p-amino- $\frac{\bar{c}}{\bar{c}}$. salicylic acid in tuberculosis, and the combined $\vec{\nabla}$ effects of quinine and pamaquin in the radical $\propto$ cure of benign tertian malaria also illustrate the same point.

However, as Dr. Brownlee has described, there is experimental evidence to show that antibiotics $\vec{\sigma}$ may actually antagonize each other. For ex-흘 ample, a bacteriostatic substance such as aureo- 3 mycin may reduce the effectiveness of penicillin. The suggested explanation is that penicillin is $\underset{\omega}{\infty}$ bactericidal and acts best on rapidly multiplying organisms; if the bacterial metabolism is de- $\omega$ pressed by bacteriostatic agents the rate of $\mathcal{W}^{\mathrm{G}}$ multiplication is slowed and the effectiveness of 0 penicillin is thereby decreased. It is not established that such an action would occur in human ${ }_{-}$ infections, but it is certainly best not to give more $\frac{\bar{\sigma}}{5}$ than one antibiotic at a time.

\section{Cost of Chemotherapy}

The striking differences in the cost of treatme with the various drugs have been well stressed by Drs. Joules, Kilpatrick and Maclean. It is most desirable that adequate therapeutic trials should be done to establish the relative merits of $\stackrel{\perp}{\square}$ the cheaply produced sulphonamides and the ex- $\overrightarrow{\overrightarrow{0}}$ pensive newer antibiotics in the treatment of 3 infections susceptible to both groups of drugs.

\section{Administration and Dosage}

To produce its actions a drug must gain access to those parts of the body where its effects are re- $\frac{3}{3}$ quired. In most instances we rely on the bloodstream to distribute drugs in the body, but in $\frac{\mathrm{O}}{3}$ others we apply drugs directly to the desired site of action. Thus the problems of administration 9 and dosage depend ultimately on factors such as $\frac{D}{0}$ the absorption of the drug from the alimentary tract or site of injection, its distribution in the $N$ blood and body fluids, its diffusion into body $N$ cells, the metabolic changes undergone by the drug and, finally, the route of elimination from ${ }_{\sigma}^{\omega}$ the body.

\section{Absorption}

(I) Alimentary Tract

The drugs used in chemotherapy are absorbed $\frac{0}{\circ}$ to different degrees and at different rates from the $\frac{0}{\Phi}$ alimentary tract. After administration by mouth $\stackrel{\mathbb{D}}{\mathbb{D}}$ 
most of the sulphonamides are absorbed quickly and fairly completely, but only about 25 per cent. of sulphaguanidine and about 5 per cent. each of succinylsulphathiazole and phthalylsulphathiazole are absorbed. Chloramphenicol and aureomycin are both well absorbed when given by mouth and this is their usual route of administration. Penicillin is absorbed irregularly even when the destructive action of gastric $\mathrm{HCl}$ is prevented by giving antacids, and oral administration of even five to ten times the injected dose is not consistently satisfactory. However, this route is justified in young children in whom injections are undesirable. Streptomycin is absorbed hardly at all from the alimentary tract, but it is not destroyed and can therefore act upon susceptible microorganisms in the lower bowel.

\section{(2) Sites of Injection}

After intramuscular injection the rate of absorption depends on the nature of the compound and the composition of the medium in which the drug is dissolved or suspended. This problem was at one time much discussed in relation to the various preparations of bismuth used in the treatment of syphilis, but is now of greatest interest in connection with penicillin. With watery solutions of sodium penicillin II, absorption is rapid and high blood levels are produced, reaching a peak in $\mathrm{I}_{5}$ to 30 mins. The duration of an effective blood level naturally depends on the dose, but according to Garrod is about 3 hours after 50,000 units and $7 \frac{1}{2}$ hours after 500,000 units of penicillin. When allowances are made for the time needed for bacteria to recover from the actions of penicillin it is probable that 50,000 units are effective for 4 hours and 500,000 units for 12 hours.

After intramuscular injection of 300,000 units of a suspension of procaine penicillin in oil or water, absorption of active penicillin is greatly delayed so that the peak blood concentration ( $\mathrm{I}$ unit $/ \mathrm{ml}$.) is reached only after 4 hours, but a detectable blood level persists for about 24 hours. The addition of aluminium monostearate delays absorption still further, and when 600,000 to $1,200,000$ units are given penicillin is present in the blood for several days.

For the treatment of acute infections watery solutions of sodium penicillin II are most satisfactory, but in the treatment of syphilis a maintained low blood concentration of penicillin is best, and therefore procaine penicillin+aluminium monostearate in oil is the preparation of choice.

Intravenous injection of drugs for chemotherapy is useful in those cases where one wants to produce a high blood level as quickly as possible (e.g. sodium sulphadiazine in meningococcal meningitis) or where a drug is too irritant to give by other routes of injection (e.g. organic arsenicals in syphilis).

The high blood levels which follow intravenous injection of a drug may sometimes be toxic to the heart. Emetine, which may cause cardiac arrhythymias and other signs of cardiac damage, should therefore never be given by this route. Intravenous administration is sometimes very wasteful, as in the case of drugs which are excreted rapidly via the kidney, and for this reason penicillin is not so given today.

\section{Distribution}

The distribution of drugs via the bloodstream is usually very satisfactory but certain regions of the body are not very accessible. For example many drugs do not penetrate at all well into the cerebrospinal fluid (or into the intraocular fluids), Penicillin appears in normal C.S.F. only in traces, and even in meningitis the amounts are quite inadequate to control an infection due to a susceptible organism such as the meningococcus. Streptomycin passes from the blood to C.S.F. more readily than penicillin, but to be effective in the treatment of meningitis, both these drugs must be administered directly into the C.S.F. Sulphonamides pass into the C.S.F. much more easily and for this reason they are the drugs of choice (sulphadiazine is best) in the treatment of meningococcal meningitis. Chloramphenicol enters the C.S.F. and intraocular fluids more readily than does aureomycin.

Diffusion of drugs into abscesses, pleural effusions and infected joints is also poor.

Occasionally a drug becomes greatly concentrated in a particular organ of the body. For example, Dr. Adam. refers to the fact that the antimalarial drug, chloroquine, becomes so much concentrated in the liver that it is effective in the treatment of hepatitis due to Entamoeba histolytica, the much lower concentrations found in the blood being quite ineffective against the acute infection of the large bowel. Certain esters of penicillin (e.g. 'Estopen') which are excreted in high concentrations in sputum are for that reason useful in the treatment of suppurative chest conditions.

Where distribution via the blood stream is inadequate local applications may be necessary. For example, in the eye it is very difflcult to produce antibacterial concentrations of penicillin by systemic administration, but subconjunctival injection of the drug produces highly effective levels in the anterior chamber. With local applications to mucous surfaces and wounds the chief problem is to ensure adequate penetration for sufflcient length of time to all parts of the area. This is best achieved by applying the drug in the form of a powder or suspension. 


\section{Fate of Drugs}

Drugs, including antibiotics, are chemical compounds of known composition which undergo metabolic changes in the body. Sometimes these changes produce the active antibacterial agent. For example, the original inactive Prontosil Rubrum is converted in the body to the active sulphanilamide. Ehrlich's famous 'Salvarsan' is oxidised to the much more active oxophenarsive (mapharsen) and proguanil is activated to a compound of at present unknown composition.

Metabolic breakdown products of drugs are usually therapeutically inactive, but sometimes they may be toxic. The well-known insolubility of certain acetylated sulphonamides will be recalled in this connection.

\section{Excretion}

Most chemotherapeutic drugs appear in the bodily secretions. Their presence in bile might lead one to suppose that they would be effective in the treatment of biliary tract infections; unfortunately this is usually not the case as any infection which damages the liver greatly reduces the concentration of drug in the bile.

Excretion is mostly via the kidney and the concentration of drug in the urine greatly exceeds the blood level. This means that even though the drug be largely inactivated in the body (e.g. chloramphenicol and aureomycin), effective antibacterial concentration still occur in the urine. Moreover, in the case of penicillin which is excreted mainly by the renal tubules, the rate of excretion can be greatly decreased by certain subtances which selectively depress renal tubular function (caronamide, benemid). In this way higher blood penicillin levels can be attained.

It is often said that owing to the high concentrations which appear in the urine, sulphonamides may be given in smaller doses for the treatment of urinary tract infections. However, since the infection is often a pyelonephritis some of the organisms are only accessible via the blood stream, so that ordinary dosage might be required.

\section{Dosage}

The principles of dosage are simple enough. First a large loading dose is required to produce an adequate drug concentration in blood and body fluids as soon as possible, and this is followed by smaller maintenance doses at such intervals as will sustain the required levels until the infection is brought under control. In nearly all cases the drug should be stopped when the temperature has been normal for three days, and usually treatment with these drugs should not exceed a week. If administration is prolonged the incidence of toxic effects is greatly increased, and the developmen of drug resistant organisms might occur.

\section{Toxic Effects}

The parasiticidal action of drugs cannot be ex pected to be so specific that no harm whatever is done to the host. It is always a question of the margin between the therapeutic and toxic doses? and in some cases this is very small, or the thera peutic and toxic ranges may even overlap. At the present day, when there may be several drugsీ which can kill off a particular organism, the mose valuable will clearly be the least toxic member of the series. For this reason penicillin has replaced the organic arsenicals in the treatment of syphilis and the older sulphonamides, e.g. sulphapyridrinę have been supplanted by compounds such as sulphamerazine and sulphadimidine in the treativ ment of bacterial infections. Thus, many toxi actions may nowadays be avoided by using a morE్ specific, less toxic compound and this is the firsti and most satisfactory way of tackling the problenor of drug toxicity.

The occurrence of nausea and vomiting is sometimes very troublesome with drugs given orallye It occurred particularly with sulphapyridine but i⿸ much less frequent with the newer sulphonamides:P.A.S., chloramphenicol and aureomycin are sisg liable to irritate the alimentary tract. To avid gastric irritation a drug should either be givetêtion enteric coated capsules which do not disintegrate in the stomach (e.g. emetine bismuth iodide) of tablets should be crushed and taken with a fer ounces of water or milk so that the substance passes rapidly through the stomach. If the dru항 causes vomiting solely by peripheral irritation injection of the drug may avoid this complication but some drugs (e.g. digitalis and sulphonamides) excite vomiting by both peripheral and centrais mechanisms, whereas others, e.g. apomorphine. and streptomycin, appear to act centrally.

\section{Toxic Effects on the Nervous System}

Peripheral nerve degeneration has been seeñ following the administration of a number of drugs, and was rather frequent with some of the earlier sulphonamides. The most important. nervous complications in chemotherapy today are the eighth nerve changes produced by strepto. mycin and dihydrostreptomycin. Streptomycis acts predominantly to depress vestibular function? though larger doses cause deafness as well; con versely dihydrostreptomycin, originally claimed be less neurotoxic than streptomycin, acts on thes auditory mechanism before it affects vestibular function, and since its effects are irreversible oit should never be used. The vestibular damage dute to streptomycin depends on the dose, which is the 
reason why the daily dosage of this drug in tuberculous infections has been reduced from $2 \mathrm{~g}$. daily to I g. daily. In such a grave condition as tuberculous meningitis it is, however, necessary to give $2 \mathrm{~g}$. intramuscularly $+100 \mathrm{mg}$. intrathecally daily, and it is not surprising that vestibular damage occurs quite frequently with the high concentrations of streptomycin so produced (see Dr. Buxton's article). But vestibular damage is often reversible and compensatory mechanisms can develop.

\section{Sensitization Reactions (Drug Allergy)}

Drug rashes (e.g. maculopapular, urticarial) and drug fever (alone or with rashes) are the commonest manifestations of drug sensitization or allergy. It is important to realize that, in patients who are having a drug for the first time, the process of sensitization usually takes five to seven days to develop. Therefore if the course of drug administration is completed within this time the liability to sensitization reactions is greatly reduced. Of course, in patients who have previously become sensitized to a particular drug, the very first administration of a second course may evoke the reactions of sensitization, as has happened with somé of the sulphonamides, especially sulphathiazole. It is worth remembering that very occasionally serious, and indeed fatal, complications such as periarteritis nodosa as well as focal necroses in the myocardium, bone marrow, spleen and liver and kidney may follow administration of the sulphonamides. The cummonest complication with penicillin is urticaria, often with extensive oedema as in 'serum sickness.' Although the number of reported cases is so far small it already appears that chloramphenicol and aureomycin can cause drug fever and rashes.

Another sign of sensitization is the development of ' contact dermatitis' from local applications to the skin, particularly if the drug is applied to an abraded surface. This has occurred quite frequently with sulphonamides, and also with penicillin, but sensitization of this type occurs most easily with streptomycin, and workers employed in its manufacture, and nurses who are concerned with its administrition, are very liable to develop dermatitis, unless appropriate precautions are taken.

Many would include among the reactions of sensitization the bone marrow reaction which leads to granulocytopaenia or agranulocytosis. This rare complication has been associated particularly with sulphonamides, and does not occur after penicillin (which indeed is used in its treatment), but it is not yet known whether it can be elicited by the newer antibiotics. Agranulocytosis usually develops only after prolonged treatment (e.g. two weeks or more) with full doses of the offending drug, and the risk of its occurrence is another indication for keeping the period of treatment as short as possible.

Thus it is most important to take account of these sensitization reactions in assessing the significance of rashes or fever which develop during chemotherapy. If the fever due to the original infection has subsided and the patient has had a normal temperature for two days, a sudden rise of temperature might be due to a recrudescence of the infection, to drug fever or perhaps to agranulocytosis. It is important to consider this question carefully as the remedy for the two latter states is so simple.

Nowadays, when there is often a choice of drugs for the treatment of a particular infection, a patient who has becom sensitized to one drug should tolerate another. For example, a patient who has become sensitized to sulphonamide might well be sensitive to other drugs of this group. Thus, a patient known to be sensitive to a drug, even though it be the first choice for a particular infection, should be treated with a drug belonging to a different chemical group. This is one of the great advantages of having more than one drug for the treatment of a given infection.

\section{Disturbances in Bacterial Flora}

Among the toxic effects which sometimesô accompany chemotherapy are those which may be? due to the indiscriminate destruction of commensals as well as pathogenic micro-organisms. It is sometimes desirable to sterilize the alimentary tract, as, for example, before operations on the bowel which might contaminate the peritoneum, but there is evidence which suggests that glossitis, angular stomatitis, and inflammation in the rectum and vagina, may often be due to the upheaval in bacterial ecology produced by chemotherapy; this leads to the unusual predominance of such insensitive organisms as B. coli and monilia such as Candida albicans. Some of the changes, e.g. angular stomatitis have been attributed to deficiency of vitamin B complex, e.g. ariboflavinosis, but administration of vitamin B does not always prevent or relieve these complications, and the abnormal flora might well act directly to cause such changes.

The flatulence and looseness of the bowels which occur with chloramphenicol and aureomycin may also be due to alterations in the flora of the alimentary tract, but direct irritation by the drugs might sometimes cause diarrhoea in the same way as it may produce vomiting.

The future of chemotherapy is difflcult to forecast, but some of the long-term problems are beginning to be revealed. The gradual development of drug-resistance of certain bacterial 
species is a striking illustration of the adaptive power of living organisms to their environment and how far this process will eventually nullify the benefits which chemotherapy has provided, or how far the dislocations of bacterial ecology will perhaps prove harmful to man, are questions which no one can answer. On the hopeful side there are of course the possibilities of gaining control of infections due to viruses and a beginning has been made in this field with the introduction of chloramphenicol and aureomycin.

In conclusion, one might recall how all the fundamental factors involved in modern chemotherapy were outlined, and in many cases thoroughly studied, by Ehrlich in the early years of this century. His practical achievements were perhaps less important than his establishment of the principles of the subject. His 'chemoreceptor' theory, with the recognition of 'parasitotropic' and 'organotropic' effects of drugs, led to the introduction of what is now called the chemotherapeutic index, which Ehrlich defined as the ratio between the 'dosis toxicata' and the 'dosis tolerata.' According to Ehrlich the aim of chemotherapy is to produce "Therapia sterilisa? magna,' that is, cure with one large sterilizing dose of the drug. This can now be done with penicilin in acute gonorrhoea, but is not yet attainable with other infections.

Ehrlich was particularly interested in the distribution of drugs in the body, and indeed earliest work dealt with the selective uptake of dyes by different tissues. Throughout his life fe worked with dyes, and all the important drugs which he discovered were coloured substancss which had a selective affinity for pathogenic parasites. Much of his work consisted of studies in the synthesis of dyestuffs in the test tube and the testing of their staining properties on cotton threads. Martha Marquardt, who was Ehrlicls secretary for many years, tells us in her book 'Paul Ehrlich' that he loved bright colours, 'the sight of a bunch of gay colours would make hich quite ecstatic.' It is thus perhaps not too fanciful to say that the structure of modern chemotherapy has been built on foundations which were createg from a highly developed responsiveness to colour in a scientist who happened to be a genius.

\section{NOTES}

\section{PETHIDINE IN ANAESTHESIA}

The increasing popularity of pethidine to potentiate nitrous oxide-oxygen anaesthesia and allow it to be used in major surgery, has prompted Burroughs Wellcome \& Co. to issue a special strength of ' Wellcome' brand Injection of Pethidine Hydrochloride for this purpose. The new strength is I per cent. and is additional to the 5 per cent. strength. It is issued in rubber-capped bottles of $25 \mathrm{cc}$. (4/6 subject). In order to avoid confusion between the two strengths, each pack will be suitably over-printed in red figures. In addition, the new product will bear a black rubber cap as a contrast to the yellow one used on the existing 5 per cent. solution. The advantages of the I per cent. solution in anaesthesia are that it can be measured easily to an accuracy of I mgm.. that dilutions are not required and that risk of thrombosis is reduced to a minimum.
CHOLINVEL-THE V. L. CHOLINE SYRU용

An agreeable liquid preparation of choline dihydrogen citrate. This has the same therapeutic action as choline chloride, but is without the disagreeable flavour. It is being used for coesditions associated with disordered hepatic fune tion, including fatty liver and cirrhosis, and कs under clinical trial for atheroma, including cases of coronary occlusion.

Prepared in a ro per cent. solution, one or the small teaspoons provide 0.35 to $0.7 \mathrm{~g}$. This concentration allows a daily dose of 1 to 3 which is that being employed for most conditionfs.

It is recommended to be given with or after meals to prevent toxic effects such as abnormal lowering of blood pressure, but much larger doses than those mentioned have been given for prolonged periods without any ill-effects, and it is thought that the dangers of toxicity may hape been over-emphasised. Available in bottles of 6 fluid ounces. 